\title{
What are the True Costs of Major Trauma?
}

\section{Submitted to \\ Journal of Trauma: Injury Infection and Critical Care}

David Rowell ${ }^{1,2,}$

Luke Connelly ${ }^{1,2,3}$

Jodie Webber ${ }^{3,4}$

Vivienne Tippett ${ }^{5,6}$

David Thiele 7,9

Michael Schuetz ${ }^{7,8}$

\section{Affiliations:}

1. School of Economics, The University of Queensland

2. Australian Centre for Economic Research on Health (ACERH)

3. Centre of National Research on Disability and Rehabilitation Medicine (CONROD)

4. IMS Health

5. Australian Centre for Pre-hospital Research, The University of Queensland

6. Queensland Ambulance Service

7. Queensland Health (The Princess Alexandra Hospital)

8. Queensland University of Technology

9. The University of Queensland

Email: d.rowell@griffith.edu.au

Citation: Rowell, D., Connelly, L.B., Webber, J., Tippett, V., Thiele, D. \& Schuetz, M., (2011) 'What are the true costs of major trauma?' Journal of Trauma: Injury Infection and Critical Care, 70(5), 1086-1095

Funding provided by the Royal Australasian College of Surgeons for the 2006 Trauma Fellowship.

Preliminary work presented as: Multiple Trauma Patients - What are the real costs for society?, The $78^{\text {th }}$ Annual Scientific Conference of the Royal Australasian College of Surgeons, The Convention Centre, $6^{\text {th }}-9^{\text {th }}$ May 2009, Brisbane, Queensland. 


\section{INTRODUCTION}

It is well-known that injury is the primary cause of death in Australians under 45 years of age, is a leading cause of all-ages mortality, morbidity and permanent disability, and is a major source of health care costs.(1) The Australian public health literature contains a number of studies of the cost of injury. Several studies have estimated the cost of specific injuries (e.g., spinal cord injury $(2,3)$ and hip fractures $(4,5)$ while others have focused on costs attributable to a particular external cause of injury (e.g., road trauma(6,7) or falls(8-10)). Only a few evaluations however, have reported the aggregate costs of trauma at the state(11-13) and national(1, 14) levels and fewer still have examined major trauma.(12)

The methodologies used to report the aggregate costs of trauma have varied. Watson, Ozanne-Smith and Moller adopt a broad focus wherein the cost of any injury ${ }^{\mathrm{i}}$ resulting in death, inpatient admission or medical treatment without hospitalisation was included(13, 14), whereas Hendrie focused on injuries ${ }^{\text {ii }}$ that required a hospital admission. $(11,15)$ While some economic evaluations have focused on the direct costs of medical treatment $(1,12-14)$ others have also included the indirect costs of trauma, such as the opportunity cost of labour forgone. $(11,15)$

The purpose of this paper is to identify the costs of major trauma. Individuals who have been subject to major trauma are vulnerable and their prognosis depends, in large part, on the delivery of high quality health care services. To date only Doran et al have reported on the direct costs (pre-hospital

\footnotetext{
${ }^{i}$ An injury was defined as ICD-9-CM injury code (E800-E999), excluding medical misadventure (E870-E879) and pharmaceutical poisoning (E930-E879)

ii An injury was defined as ICD-10-AM injury code S00.0 to T98.3 or an external cause of injury code V01.0 to Y98
} 
and hospital) of major trauma ${ }^{\text {iii }}$ with an injury severity score (ISS) $>15$, in an Australian inpatient population.(12) While Doran et al have used the ISS to differentiate admissions for major trauma from less acute presentations, mean ARDRG cost weights were used to impute the costs of an inpatient admitted for major trauma. This approach would underestimate the true cost of major trauma if admissions with an ISS > 15 were more costly than the AR-DRG cost mean. This paper investigates the hypothesis that the cost of major trauma in Australia is, consequently, higher than currently-published estimates suggest.

\section{MATERIALS AND METHODS}

We focus on measuring, accurately, the direct and indirect costs associated with major trauma, including all costs that were incurred during a period of 12 months following discharge from the PAH. This period was selected somewhat arbitrarily, but constitutes an improvement over existing Australian studies, which have focused on the index admission.

The direct costs of injury can be estimated using a top-down or bottom up methodology. A top-down approach typically involves estimating the total cost of treatment and calculating the arithmetic mean. All inpatients that receive a given treatment are thus allocated an identical 'average' cost according to this approach. The alternative, bottom-up, approach generates a schedule of costs that reflects a unique set of clinical input costs which comprise the treatment received by each individual inpatient. The advantage of this approach is that it enables a more complete picture of the composition of total costs, when undertaken carefully, this can also provide some reassurance that inputs that are captured, and their costs, are indeed attributable to the care of the relevant patients.

The costs of trauma are analysed for a sample of inpatients treated at a major Australian tertiary hospital, the Princess Alexandra Hospital (PAH), which is one of two major tertiary hospitals located in Brisbane, Australia. Costs from

\footnotetext{
iii An injury was defined as ICD-CM codes 800 to 995.
} 
three data sources were matched to provide an aggregation of direct and indirect costs incurred from admission to the emergency department up to one-year post discharge, as follows.

\section{The Sample}

In 2004 PAH treated 1,976 inpatients for traumatic injury. Of this number, $336(17 \%)$ were treated for major trauma, as indicated by an New Injury Severity Score (NISS) > 15 were treated for major trauma.(16) The NISS is an anatomically based ordinal scale (1-75). Each injury is evaluated using an Abbreviated Injury Scale (AIS), which is allocated, to one of six body regions. The NISS is calculated as the sum of the squared scores for the three most acutely injured body regions, or the maximum score (of 75) if any severity AIS is equal to six ("potentially critical/unsurvivable").(17) As this is not an intervention study, our objective was to maximise the size of the sample that could be followed, subject to our resource constraints. Due to the comprehensive nature of the cost categories to be collected and the labour-intensiveness of the collection of survey data, hospital data, Medicare data, and so on, we estimated that we could approach approximately 70 people, which, assuming a response rate of approximately $50 \%$, would result in a sample size of approximately 35 people.

To create the sample frame, we applied the following inclusion criteria: (i) length of stay $($ LOS) $>24$ hours, (ii) age > 18 years, (iii) no history of mental illness, (iv) not registered with the Department of Veteran Affairs (v) NISS > 15 and (vi) Diagnosed with one of 6 Australian Refined Diagnosis-Related Groups (AR-DRG) codes for 'Multiple Significant Trauma'(18) (See Appendix 1 for the frequency of admission by AR-DRG). The inclusion criteria were applied in three steps. First, administrative data on (i), (ii), and (vi) were applied, resulting in a sample-frame of $n=250$. From that sample frame, 80 subjects were randomly drawn for inclusion in the study. Selection was undertaken using a random number generator and matching random numbers to patient identification numbers. Upon close inspection of the records of these 80 subjects, a further eight subjects were 
excluded on the basis that they did not meet the inclusion criteria (e.g., (iii), (iv), (v)). This approach resulted in a sample of 72 subjects.

\section{Cost Estimation}

Cost estimates were generated using data from three major sources: (i) the survey instrument, (ii) the PAH and (iii) Medicare Australia. In addition, we obtained data from the Queensland Trauma Registry (QTR) on traumatic injuries across the state of Queensland. These data were used to conduct comparisons between our hospital-specific sample and a state-wide sample of traumatic injuries, as well as to estimate the cost of trauma across the state.

Survey Instrument: The selected participants were mailed our survey instrument, which is henceforth referred to as the Cost of Trauma Instrument (COTI). (19) The COTI is a 20-item survey, which was developed to estimate the direct and indirect costs of major trauma incurred during the 12 months following discharge from hospital. A copy of which can be freely downloaded from the CONROD website.(19) If no reply was received within four weeks, the participants were invited to complete the survey via the telephone. The survey participation rate was $61.7 \%$. Data cleaning required the further removal of 14 respondents who were subsequently identified with an NISS $<16$ thus the final sample included 30 inpatients discharged from PAH.

The COTI data collected included, employment status, pre- and post-injury wage rates, out-of-pocket expenses and hours of informal care provided by family members. These data were used to derive three cost estimates: (a) out-of-pocket expenses for over-the-counter medications, allied health care and home renovations incurred up to one-year post discharge; (b) estimates of property damage that occurred at the time of, and in relation to, the injury; and (c) the opportunity costs of employment foregone for both the injured parties and their unpaid carers. The Human Capital Approach (HCA), which estimates the monetary value of time spent in the workforce(20), was used to estimate opportunity costs of employment foregone, up to one-year post discharge as follows: 


$$
\begin{gathered}
\mathrm{OCL}_{\mathrm{I}}=\left[\mathrm{T} * \mathrm{~W}_{\text {I pre-trauma }}\right]+\left[(52-\mathrm{T}) *\left(\mathrm{~W}_{\mathrm{I} \text { pre-trauma }}-\mathrm{W}_{\mathrm{I} \text { post-trauma }}\right) \text { Model } 1\right. \\
\mathrm{OCL}_{\mathrm{F}}=52 \mathrm{~W}_{\mathrm{F}} * \mathrm{C} \text { Model } 2
\end{gathered}
$$

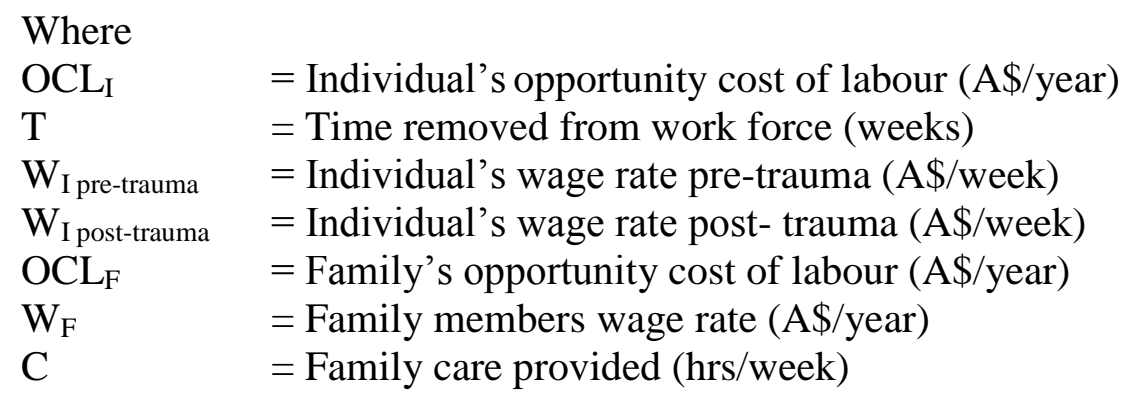

Model 1 estimates personal opportunity cost where the first term estimates the loss of income due to time absent from the workforce and the second term estimates the loss of earning capacity due to any residual disability after returning to the workforce. Model 2 estimates loss of family income as simply the product of time in the workforce foregone and stated wage rate.

Princess Alexandra Hospital: Two cost estimates were obtained from PAH. The first was derived using a bottom-up approach that is produced by a hospital accounting system called Transition $\mathrm{II}^{\mathrm{TM}}$; which was developed by Transition Systems, Inc. in the late 1980s and since acquired and supported by Eclipsys Corp. in 1999.(21) This software program integrates patient level clinical and financial data, to generate a financial cost per inpatient. Transition II performs two computational tasks. Firstly, hospital departments are categorised as either direct (clinical) or indirect (non-clinical) providers of patient care. All costs from indirect cost centres are iteratively distributed to direct cost centres according to predetermined algorithms (e.g., cleaning costs are redistributed per $\mathrm{m}^{2}$ ). Secondly, intermediate products, including labour and material costs, delivered to patients in direct cost centres are identified and valued. A weighting procedure is employed to ensure the valuation of intermittent products does not involve a double counting of costs.(22)

A chart review was conducted to ensure that supplemental treatments related to the initial admission for trauma were identified and included in the economic evaluation. The Transition II system produces estimates of the treatment 
costs as follows. The costs of emergency treatment are generated using an algorithm that is based on both triage and disposal status. Inpatient costs (medical, ward and ICU costs) were estimated using an hourly cost of care. Inpatient clinical services (Pathology, Pharmacy, Prosthetics, Radiology and Allied Health) were estimated using an average cost per service. Operating theatre costs (pre-, intraand post-op) were estimated using LOS measured in minutes. The costs of outpatient services were estimated using an average cost per service. Note that our use of these cost data implicitly assumes that marginal and average costs of treatment are equal.

A second set of cost estimates, obtained via a top-down approach, used the Queensland Health Funding Model (QHFM) to estimate the cost of treatment. The QHFM is used by the Queensland Heath Department to determine revenue remitted to PAH for inpatient treatments. A notional estimate of inpatient costs was derived using an AR-DRG mediated algorithm. Where LOS was above or below pre-determined trim points (which are used to identify LOS outliers), a per diem adjustment to the revenue received was made. A further adjustment of revenue remitted was determined by the utilisation of outpatient services. Emergency department costs were estimated using the patient's triage and discharge status. Outpatient clinic costs were estimated by obtaining an average cost per visit, and using this as the best available estimate of the marginal cost of treatment.

Medicare Australia: We obtained consent from all respondents to extract data on all fee-for-services medical care provided in- and out-of-hospital, plus pharmaceuticals consumption as recorded by Medicare Australia. Specifically, Medicare Australia provided an itemised list of all medical and pharmaceutical services, by subject, which includes all services that are subsidised under the Medicare Benefits Scheme (MBS) and Pharmaceutical Benefits Scheme (PBS) for the 12-month period following the initial inpatient discharge.

Figure 1 provides a schematic that illustrates our costing methodology. 


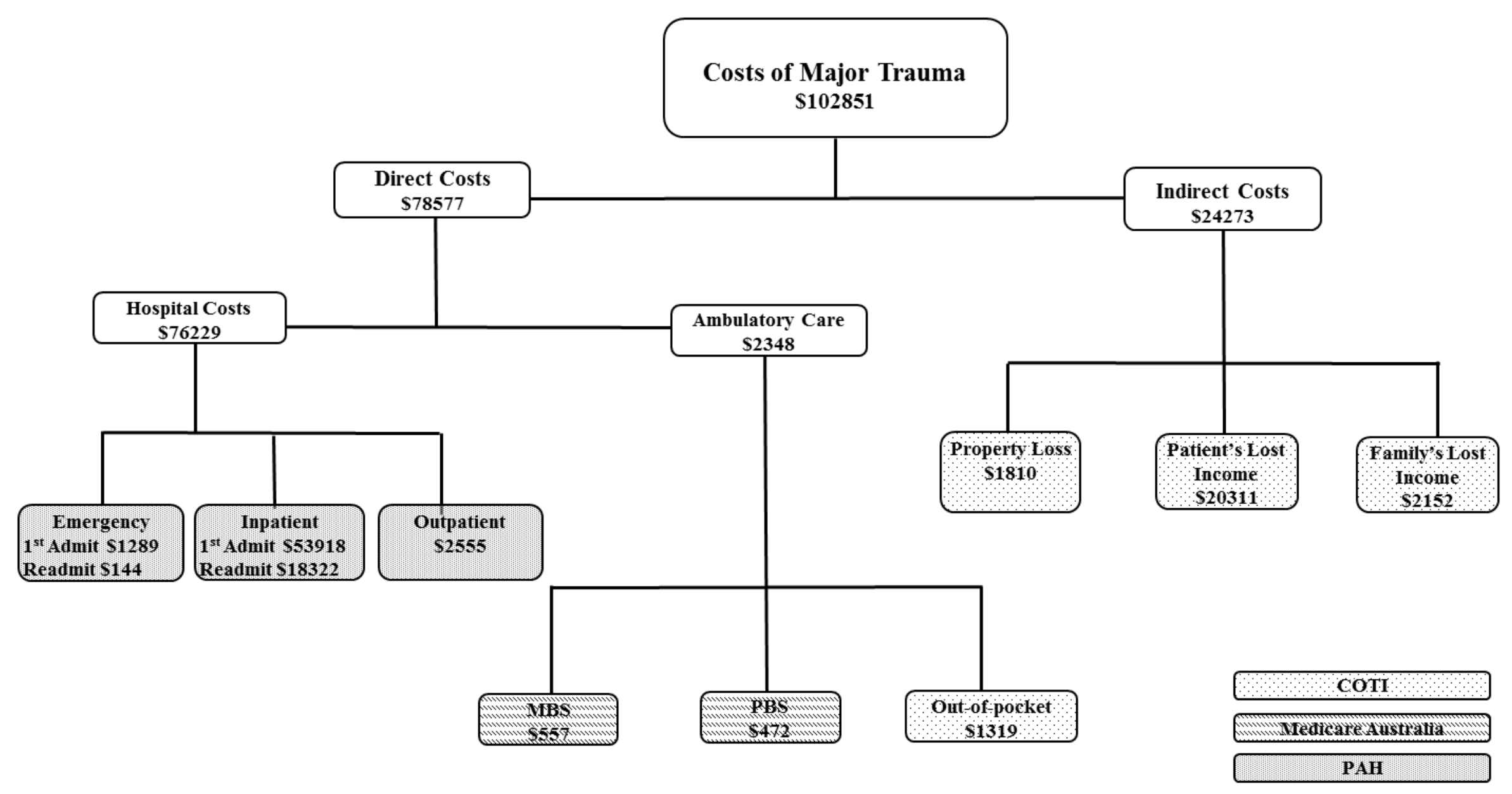


Our methodology reports an inventory of costs incurred in 2004-05 by a sample of 30 discharged inpatients. A naive estimate of the total costs of major trauma could simply be given by the product of the number of treated inpatients and average cost per patient. However given the small sample size $(n=30)$ we improve the external validity of our results, by exploiting inpatient population data obtained from the QTR. In 2004, the QTR collected data from 12 major public hospitals in Queensland, which increased to 13 public hospitals in 2008. An examination of Queensland Health's Quarterly public hospitals performance report (23) for 2009 reveals that those public hospitals, which contributed to the QTR (16), accounted for 59.3\% of all emergency department attendances in Queensland and $85.1 \%$ of emergency department attendances to the larger and specialised hospitals. We therefore believe that collectively these hospitals admit a near complete population of inpatients treated for major trauma in Queensland. The inclusion of data from the QTR serves two purposes. Firstly, it enables our sample characteristics to be benchmarked against PAH and state-wide trauma results. Second, we exploit QTR data to estimate the costs of treating major trauma for 2004 and 2008.

Our inpatient sample was used to estimate two models of cost.

$$
\begin{array}{r}
\text { Cost }=\alpha_{0}+\alpha_{1} L O S+\alpha_{2} I S S+\varepsilon_{i} \\
\text { Model } 3 \\
\ln \text { Cost }=\beta_{0}+\beta_{1} L O S+\beta_{2} I S S+\eta_{i} \\
\text { Model } 4
\end{array}
$$

In Model 3, the dependent variable measure Cost is a measure of total costs incurred. The independent variables include $L O S$ for length of stay in hospital (measured in days) and an index of patient acuity, the ISS, is the injury severity score. Stevenson et al. (24), have argued that ISS should be treated as a categorical variable. They produce a frequency distribution to illustrate that the ISS values peak at values 1, 4 and 9 and suggest that the categorical variables would be preferable.(24) However, the frequency distribution of ISS from values 15 to 75 decreases monotonically. Since all observations in our sample had an ISS > 15, we 
treat NISS as a continuous variable. In Model 4, we estimate the log of costs $\ln$ Cost to control for possible bias due to heteroscedasticity in the error term. The coefficients derived from Models 3 and 4 were used to estimate the costs of major trauma using population data which included LOS and ISS for all admissions for major trauma supplied by the QTR for the years 2004; PAH $(n=326)$ and Queensland $(n=1300)$ and 2008; PAH $(n=440)$ and Queensland $(n=1832)$.

This method requires that the sample coefficients for LOS and ISS provide an unbiased estimate of the population coefficients. As the population parameters are unknown, it is not possible to verify the validity of this assumption. However, by comparing the relationship between ISS and LOS for the sample with the QTR populations the representativeness of our sample can be assessed. We therefore estimated the following univariate specification $L O S=\alpha_{0}+\alpha_{1} I S S+\varepsilon_{i}$ for our sample and four QTR populations of inpatients treated for major trauma, PAH and Queensland for the years 2004 and 2008. The dependent variable, LOS, is as continuous variable and therefore the univariate specification was estimated with OLS regression. A sensitivity analysis was also conducted though, in which LOS was treated as a count (of days), and a Poisson regression was estimated. Wald tests were utilised to determine if there were differences between the ISS coefficients for the sample and each of the QTR populations i.e. $H_{0}:\left(\right.$ Sample_$\left.\alpha_{1}\right)$ - $\left(\right.$ Population_ $\left.\alpha_{1}\right)=0$. These results were used to temper the interpretation and extrapolation of our cost estimates.

\section{RESULTS}

The average age of the sample was 37.2 years, mean LOS was 32 days and mean NISS was 28.13 . Females comprised $13.3 \%$ of sample. The mean AR-DRG cost-weight (25) (a linear indicator of resource intensity) was 11.7. The mean cost of treating an inpatient with an AR-DRG cost-weight equal to one in 2004, was A $\$ 2,985$.(25) If the focus were all inpatients treated for trauma, as opposed to the sub-set of inpatients treated for major trauma, a cost weight of 11.7 would imply an average cost of $\mathrm{A} \$ 34,925$. 
An important question is whether our sample of major trauma inpatients is representative of the population of trauma inpatients treated at the PAH. Table 1 reports the sample means alongside reference populations of inpatients treated for major trauma at the PAH and in the State of Queensland, generally, during 2006. These data show no differences in means at the $95 \% \mathrm{CI}$, providing no evidence that our sample systematically differs significantly from the population of major trauma cases in this State.

Table 1 Sample Characteristics Contrasted with Inpatients Populations Treated for Major Trauma at PAH and Queensland.

\begin{tabular}{lcc|cccc|cccc} 
Variable & $\begin{array}{c}\text { Sample (2004) } \\
(\mathbf{n = 3 0 )}\end{array}$ & $\begin{array}{c}\text { PAH (2004) } \\
(\mathbf{N = 3 2 6 )}\end{array}$ & $\begin{array}{c}\text { QLD (2004) } \\
\mathbf{( N = 1 3 0 0 )}\end{array}$ & $\begin{array}{c}\text { PAH (2008) } \\
(\mathbf{N = 4 4 0 )}\end{array}$ & $\begin{array}{c}\text { QLD (2008) } \\
(\mathbf{N = 1 8 3 2})\end{array}$ \\
\hline \hline & Mean & S.D. & Mean & S.D. & Mean & S.D. & Mean & S.D. & Mean & S.D. \\
Length of stay (days) & 32.40 & 47.06 & 23.22 & 28.95 & 17.40 & 21.61 & 19.83 & 26.87 & 16.44 & 21.61 \\
Age (years) & 37.20 & 17.95 & 42.66 & 21.82 & 41.18 & 23.48 & 43.11 & 20.45 & 43.49 & 23.84 \\
Injury Severity Score & 28.13 & 9.92 & 25.44 & 9.98 & 24.32 & 9.86 & 24.78 & 9.04 & 24.47 & 8.82 \\
Female (\%) & 13.33 & 34.57 & 20.55 & 40.47 & 26.00 & 43.88 & 25.00 & 43.35 & 27.24 & 44.53 \\
Cost weight (CW) & 12.27 & 9.06 & $7.88^{*}$ & $6.58^{*}$ & $7.60^{*}$ & $6.74^{*}$ & 7.65 & 6.47 & 7.6 & 6.86
\end{tabular}

Table 1 Sample Characteristics Contrasted with Inpatients Populations Treated for Major Trauma at PAH and throughout Queensland.

Source: Statistics for PAH and Queensland, 2004 and 2008 were provided by the Queensland Trauma Registry (QTR).

Note: 2006 data is highlighted with an *. The QTR does not provide ARDRG cost weights. However, in 2006 the QTR reported operation-type, for the first time. This information was combined with ICD-10-AM codes indicating injury type (S00-S99, T00-T35, T6, T66-T71 or T75) to classify inpatients by one of the six AR-DRG V5.0 codes for Multiple Significant Trauma(18) and then matched to the appropriate cost weight.(25) Thus last row in Table 1 compares the mean costs weights for a sample of inpatients treated at PAH $(n=30)$ in 2004 with inpatients populations treated for major trauma at PAH $(\mathrm{N}=379)$ and QLD $(\mathrm{N}=1733)$, in 2006.Table 2 reports a bottom-up estimate of the costs of major trauma treated in a sample of 30 inpatients discharged from PAH in 2004. The second column reports the mean cost of the initial presentation to $\mathrm{PAH}$. The third column presents the mean costs of all subsequent, clinically related presentations, 
as determined by expert medical review, to PAH that occurred during 2004. The fourth and fifth columns present mean costs of all treatments received and their standard deviations while the sixth column presents median costs. The final column provides an indicator of the approach that was used to derive each cost estimate.

Table 2 Costs of major trauma treated at PAH, 2004-05 using the "bottomup" approach A\$ 


\begin{tabular}{|c|c|c|c|c|c|c|}
\hline Inpatient Cost Categories & Initial Rx & Additional Rx & All $\mathbf{R x}$ & Std. Dev. & Median & Costing Methodology \\
\hline \multicolumn{7}{|l|}{ Inpatient Hospital Costs } \\
\hline Emergency Department (inpatient) & $\$ 79$ & $\$ 0$ & $\$ 79$ & $\$ 131$ & $\$ 0$ & Per triage \& disposal status \\
\hline Medical & $\$ 10,380$ & $\$ 8,436$ & $\$ 18,816$ & $\$ 32,910$ & $\$ 5,352$ & LOS \\
\hline Ward & $\$ 5,079$ & $\$ 1,822$ & $\$ 6,901$ & $\$ 11,638$ & $\$ 3,781$ & LOS \\
\hline $\mathrm{ICU}$ & $\$ 17,753$ & $\$ 0$ & $\$ 17,753$ & $\$ 25,512$ & $\$ 5,385$ & LOS \\
\hline \multicolumn{2}{|l|}{ Inpatient Clinical Service Costs } & $\$ 10,257$ & $\$ 43,548$ & $\$ 60,369$ & $\$ 22,622$ & \\
\hline Pathology & $\$ 2,035$ & $\$ 196$ & $\$ 2,231$ & $\$ 3,588$ & $\$ 991$ & Per item \\
\hline Pharmacy & $\$ 1,117$ & $\$ 732$ & $\$ 1,848$ & $\$ 3,604$ & $\$ 633$ & Per item \\
\hline Theatre & $\$ 2,533$ & $\$ 414$ & $\$ 2,948$ & $\$ 4,567$ & $\$ 1,102$ & Per hr; Pre-, Intra \& Post-op \\
\hline Anaesthetics & $\$ 2,804$ & $\$ 237$ & $\$ 3,042$ & $\$ 4,526$ & $\$ 977$ & Per hr \\
\hline Prosthetics & $\$ 3,507$ & $\$ 487$ & $\$ 3,994$ & $\$ 6,317$ & $\$ 316$ & Per item \\
\hline Radiology & $\$ 2,203$ & $\$ 251$ & $\$ 2,454$ & $\$ 2,198$ & $\$ 1,713$ & Per item \\
\hline Allied Health & $\$ 2,430$ & $\$ 2,720$ & $\$ 5,151$ & $\$ 8,343$ & $\$ 1,863$ & Per item \\
\hline Other & $\$ 3,998$ & $\$ 3,028$ & $\$ 7,026$ & $\$ 11,425$ & $\$ 2,658$ & Per item \\
\hline Total Inpatient Clinical Service Costs & $\$ 20,628$ & $\$ 8,065$ & $\$ 28,692$ & $\$ 35,499$ & $\$ 17,595$ & \\
\hline Total Inpatient Costs & $\$ 53,918$ & $\$ 18,322$ & $\$ 72,240$ & $\$ 94,712$ & $\$ 41,311$ & \\
\hline \multicolumn{7}{|l|}{ Clinical Services in ED on Admission } \\
\hline Emergency Department (non-inpatient) & $\$ 534$ & $\$ 13$ & $\$ 546$ & $\$ 208$ & $\$ 508$ & Per Rx \\
\hline Pathology & $\$ 143$ & $\$ 3$ & $\$ 146$ & $\$ 124$ & $\$ 108$ & Per item \\
\hline Radiology & $\$ 612$ & $\$ 10$ & $\$ 622$ & $\$ 686$ & $\$ 309$ & Per item \\
\hline Total Clinical Services in ED on Admission & $\$ 1,289$ & $\$ 25$ & $\$ 1,314$ & $\$ 730$ & $\$ 1,122$ & \\
\hline \multicolumn{7}{|l|}{ Clinical Services in ED post Admission } \\
\hline Emergency Department (non-inpatient) & n.a. & $\$ 101$ & $\$ 101$ & $\$ 246$ & $\$ 0$ & Per Rx \\
\hline Pathology & n.a. & $\$ 4$ & $\$ 4$ & $\$ 14$ & $\$ 0$ & Per item \\
\hline Pharmacy & n.a. & $\$ 2$ & $\$ 2$ & $\$ 7$ & $\$ 0$ & Per item \\
\hline Radiology & n.a. & $\$ 13$ & $\$ 13$ & $\$ 36$ & $\$ 0$ & Per item \\
\hline Total Clinical Services in ED post Admission & n.a. & $\$ 119$ & $\$ 119$ & $\$ 298$ & $\$ 0$ & \\
\hline Total Clinical Services in ED & n.a. & $\$ 144$ & $\$ 1,433$ & $\$ 787$ & $\$ 1,197$ & \\
\hline \multicolumn{7}{|l|}{ Outpatient Clinical Services } \\
\hline Nursing and Medical & n.a. & $\$ 584$ & $\$ 584$ & $\$ 1,078$ & $\$ 227$ & QHFM \\
\hline Pathology & n.a. & $\$ 46$ & $\$ 46$ & $\$ 65$ & $\$ 0$ & QHFM \\
\hline Pharmacy & n.a. & $\$ 21$ & $\$ 21$ & $\$ 62$ & $\$ 0$ & QHFM \\
\hline Radiology & n.a. & $\$ 306$ & $\$ 306$ & $\$ 561$ & $\$ 103$ & QHFM \\
\hline Allied Health & n.a. & $\$ 1,598$ & $\$ 1,598$ & $\$ 4,038$ & $\$ 93$ & QHFM \\
\hline Outpatient Clinical Services per Subject & n.a. & $\$ 2,555$ & $\$ 2,555$ & $\$ 5,054$ & $\$ 634$ & \\
\hline Total Recorded Costs per Inpatient & $\$ 55,207$ & $\$ 21,022$ & $\$ 76,229$ & $\$ 95,880$ & $\$ 43,356$ & \\
\hline
\end{tabular}


The most costly component of major trauma costs was inpatient hospital care, where the mean cost per patient was A $\$ 43,548$. Eighty-four per cent of these costs are attributable to Medical and ICU treatment. The average cost of inpatient clinical services was A $\$ 28,692$. These costs were spread across all clinical categories, with a total of $\mathrm{A} \$ 5,990$ being spent on Theatre and Anaesthetic services. Average inpatient costs were calculated to be A $\$ 72,240$. Three subcategories of Emergency Department costs were also calculable, namely: (i) costs incurred as a non-inpatient (immediately) prior to the initial admission, (ii) costs incurred as an inpatient during admission and (iii) costs incurred as a non-inpatient post discharge. In total, Emergency Department costs for treatment received, including subsequent presentations as an outpatient, cost A $\$ 2,826$ per injured patient. A further $\mathrm{A} \$ 2,555$ was incurred attending other outpatient clinics. The average cost for all treatments received at PAH was A $\$ 76,299$.

The inclusion of readmissions was found to be an important source of additional cost. From our sample of 30 inpatients, 16 (53.3\%) were readmitted in total on 31 occasions and one of whom was readmitted nine times. Appendix 2 provides a complete inventory of AR-DRG codes for readmitted inpatients. Many readmissions were for routine treatments (e.g. 10 readmissions for rehabilitation) while two readmissions were for treatment of complications due to joint and bone infection. Table 2 demonstrates that subsequent inpatient care increased direct costs by a further $38.1 \%$.

A modified 'top-down' estimate of costs is presented in Table 3 as a means of testing the main hypothesis of this paper, namely that the cost of trauma in the State of Queensland is likely to be higher than has been estimated using AR-DRG mean costs. The QHFM, which adjusts AR-DRG cost weights with LOS and Outpatient utilisation data, reports a mean cost per treated inpatient of $A \$ 59,801$. This estimate is considerably less than the bottom-up estimate of A\$76,229 reported in Table 2.

Chapko et al. used Canadian data to compare the equivalence of bottom-up and top-down cost estimates in a sample of 14,915 patients. They report that the 
average top-down cost estimate of an inpatient admission was $8.5 \%$ larger than the comparable bottom-up estimate, and that the correlation coefficient was 0.77.(26) However, it is uncertain in this instance to what extent all additional costs incurred treating inpatients with an NISS > 15 are fully captured with an adjustment for LOS and therefore our bottom-up estimate is preferred.

Table 3 Costs of major trauma treated at PAH, 2004-05 using a top-down approach \$A

\begin{tabular}{lllll} 
Variable & Mean & S.D. & Median & Source \\
\hline \hline Average Inpatient Episode Revenue & $\$ 57,000$ & $\$ 61,681$ & $\$ 47,750$ & QHFM $(04 / 05)$ \\
Pre-admission ED revenue & $\$ 733$ & $\$ 291$ & $\$ 580$ & QHFM $(03 / 04)$ \\
ED Revenue & $\$ 96$ & $\$ 272$ & $\$ 0$ & QHFM $(03 / 04)$ \\
Outpatient Clinic Revenue (ORMIS) & $\$ 1,063$ & $\$ 1,219$ & $\$ 724$ & QHFM $(02 / 03)$ \\
Outpatient Clinic Revenue (non-ORMIS) & $\$ 909$ & $\$ 2,295$ & $\$ 50$ & QHFM $(02 / 03)$ \\
Average Revenue Received & $\$ 59,801$ & $\$ 62,497$ & $\$ 49,638$ &
\end{tabular}

Source: Health Information Management Services PAH

Note: Operating Room Management Information System (ORMIS)

Table 4 combines the cost estimates reported in Table 2 with (i) cost data supplied by Medicare and (ii) cost data collected via the COTI. Medicare Australia provided an itemised list of medical services and pharmaceuticals consumed through the MBS and PBS up to one-year post-discharge. The mean costs of ambulatory and pharmaceutical care were estimated to be $\mathrm{A} \$ 557$ and $\mathrm{A} \$ 457$, respectively. These costs account for just $1 \%$ of the mean costs of treatment. Table 4 was supplemented with direct and indirect cost estimates obtained from the COTI. The inclusion of out-of-pocket expenses $(\mathrm{A} \$ 1,319)$ brings total direct costs of major trauma to $\mathrm{A} \$ 78,577$ per treated inpatient.

The indirect costs of major trauma were substantial. Prior to admission, 24 (80\%) respondents were employed while only 13 (43.3\%) were employed at the time of the interview. During the 12-months following discharge the respondents were absent from the workforce for an average of 20.8 weeks. The average pre-tax weekly income, pre- and post-admission, was reported to be $\mathrm{A} \$ 552$ and $\mathrm{A} \$ 276$, respectively. The opportunity cost of employment foregone during the 12-months 
following discharge was estimated to be $\mathrm{A} \$ 20,310$ for the subject and $\mathrm{A} \$ 2,152$ for the career. Property damage was reported to be A $\$ 1,810$ per injury. The total indirect costs of major trauma were estimated to be to $\mathrm{A} \$ 24,273$ per treated inpatient. Thus the total cost of major trauma, including all direct and indirect costs incurred up to one-year post discharge, was estimated to be $\mathrm{A} \$ 100,851$ per injured patient.

Table 4 Cost of Major Trauma Treated at PAH (2004-05) A\$

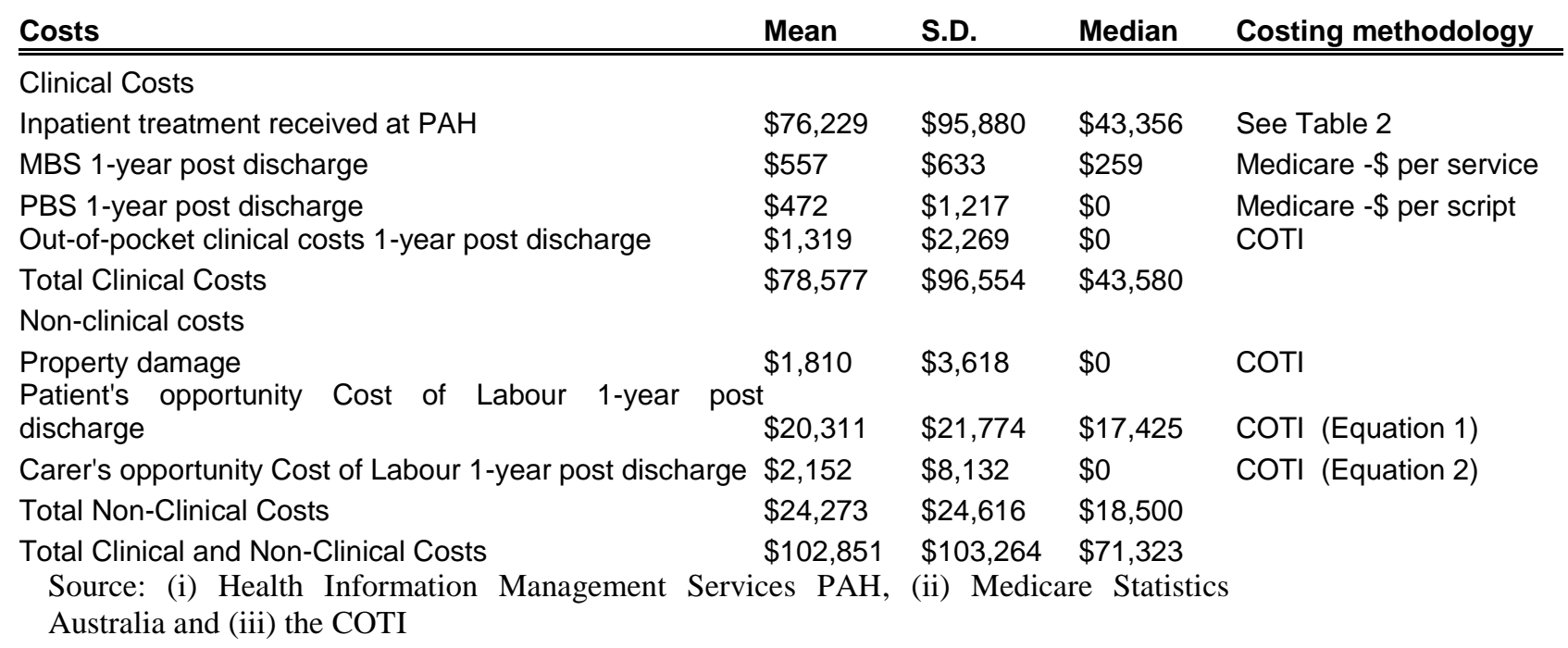

The data reported in Table 4 conform to expectations. Firstly, there is a positive correlation between NISS and cost (see Figure 2). Secondly, the cost data are left-skewed: the mean and median costs were $\mathrm{A} \$ 102,851$ and $\mathrm{A} \$ 71,323$, respectively (see Figure 3). Note the presence of two high cost inpatients in the sample. 


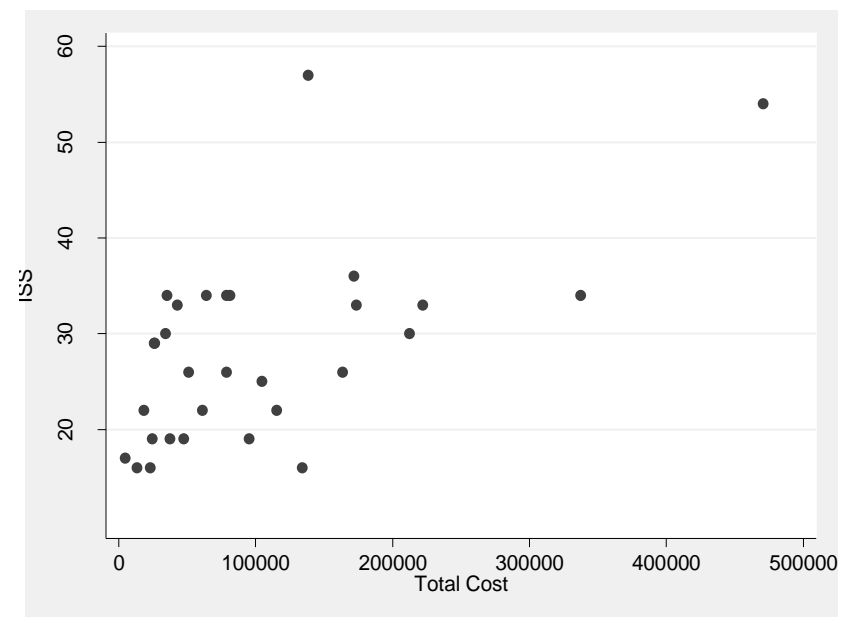

Figure 2: Correlation between NISS and total cost of major trauma treated at PAH, (20042005).

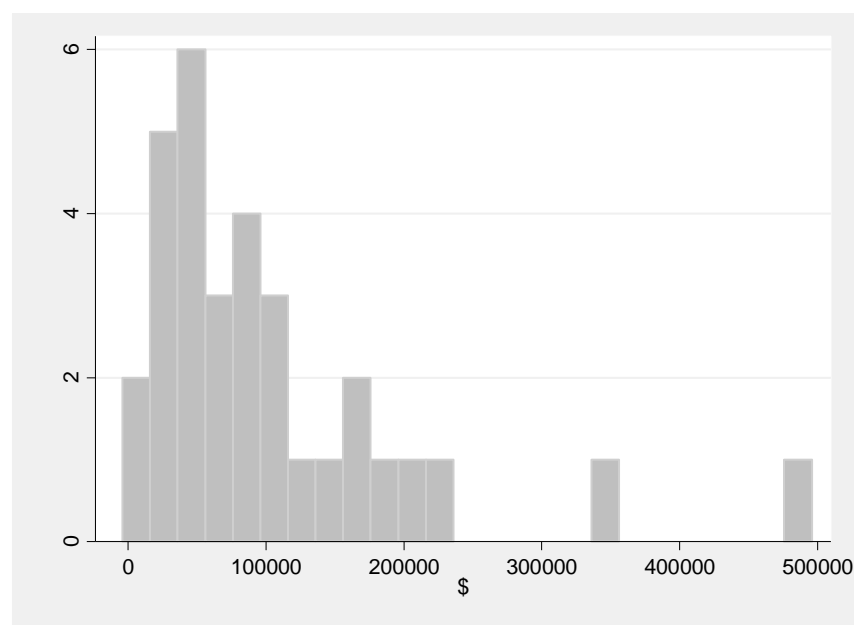

Figure 3: Distribution of the total cost of major trauma treated at PAH, (2004-2005)

The statistical parameters with $p$-values in parentheses for Model 3 were, $\alpha_{0}=14,532.38(0.633), \alpha_{1}=1,631.42(<0.01)$ and $\alpha_{2}=2,293.56(0.051)$ with an adjusted $R^{2}=0.74$. For Model 4 the estimates were, $\beta_{0}=9.833(<0.01), \beta_{1}=0.01$ $(<0.01)$ and $\beta_{2}=0.033(0.06)$ with an adjusted $R^{2}=0.48$. The reported slope coefficients on LOS and ISS have the expected positive correlation the cost of major trauma. This is interesting inasmuch as LOS and NISS are highly correlated ( $\rho=0.497$ in Model 3), but are still independently correlated with the cost of trauma. We report Shapiro-Wilk tests for normality in Model $3[\mathrm{~W}=0.93(p=$ $0.052)]$ and Model $4[\mathrm{~W}=0.976(p$-value $=0.719)]$. These results show that while 
the costs were not normally distributed in Model 3, log of costs was normally distributed in Model 4, which supports our decision to estimate this specification. A White's test for heteroskedasticity in Model $3[\chi 2(5)=1.34(p$-value $=0.93)]$ and in Model $4[\chi 2(5)=4.69(p$-value $=0.45]$ suggests the null hypothesis that the residuals are homoskedastic should be accepted. While these statistical tests suggest that these two parsimonious models provide plausible within sample estimates of the coefficients for LOS and ISS, the question of external validity remains. A battery of Wald tests is reported in the Appendix, and was used to determine if the relationship between LOS on ISS in our sample is systematically different from that of the QTR inpatient populations for 2004 and 2008. The results were mixed. While the sample coefficients for ISS were approximately two (Poisson) to 3.5 (OLS) times greater than the population coefficients, the Wald tests indicate no statistical differences between the sample coefficients and five out of the eight population coefficients, at the $95 \%$ level of confidence. These results suggest extrapolations from our sample results may be reasonable, but that some caution is required with their application.

We applied the sample coefficients for LOS and ISS to four QTR inpatient populations for major trauma to estimate patient-level cost estimates. The predicted costs summed to provide hospital and state level cost aggregates. For the state of Queensland, these estimates were inflated by a further $17.6 \%$ because the public hospitals, which contribute to the QTR, only treat $85.1 \%$ of the emergency department attendances at the larger and specialised hospitals, in the state. For 2004, Models 3 and 4 were used to estimate the total cost of major trauma for inpatient populations treated at $\mathrm{PAH}$, and in Queensland. The estimated cost of major trauma at the PAH was estimated to be A $\$ 21.2$ million (Model 3) to A $\$ 26.6$ million (Model 4) and, in the State of Queensland, A\$86.1 million (Model 3) to A \$106.4 million (Model 4). For 2008, the total cost of major trauma for inpatient populations at the PAH and the State of Queensland were estimated to be A $\$ 37.1$ million (Model 3) to $\mathrm{A} \$ 31.2$ million (Model 4) and $\mathrm{A} \$ 166.4$ million to $\mathrm{A} \$ 135$ million (Model 4). 


\section{DISCUSSION}

The strength of this partial economic analysis lies in the detailed enumeration of costs albeit conducted on modest sample of 30 inpatients discharged from a large tertiary hospital 2004. The costing methodology incorporated a bottom-up estimate of inpatient costs, including the inpatient readmissions, outpatient care as well as cost estimates for ambulatory care up to 12-months post discharge, out-of-pocket expenses and opportunity costs of employment forgone. Cost categories not incorporated in our analysis include estimates for pre-hospital transport and treatments received beyond the 12-month period of observation (e.g., rehabilitation and reconstructive surgery). Nevertheless, our costing methodology has included the most important sources of costs that are associated with major trauma.

While the primary focus of this study was to provide a detailed microanalysis of the costs of major trauma, we believe it is important to offer some perspective for these cost estimates. A naive estimate of the total costs of major trauma would simply calculate the product of the number of treated inpatients and the average cost per treated patient, e.g. A $\$ 131.2$ million $(1300 * \mathrm{~A} \$ 102,851)$ for Queensland in 2004. However, this approach assumes a representative sample. Table 1 reports mean LOS and ISS for the sample were greater than the QTR population means, however there was no statistical difference between them at the $95 \%$ level of confidence. Nevertheless, in order to make some extrapolations, for illustrative purposes, we were able to utilise actual LOS and ISS from the QTR. As the QTR is comprised of $85.1 \%$ of all emergency department attendances, at large and specialised hospitals in Queensland, we are confident that this registry provides a good approximation of the actual LOS and NISS for all inpatients treated for major trauma in Queensland.

Sample data were used to construct two statistical models to predict the cost of major trauma using two explanatory variables, LOS and ISS. The diagnostic tests $\left(R^{2}\right.$, Shapiro-Wilk \& White's test) suggest that our within-sample models of cost produce sufficient explanatory power. Caution was exercised, 
though, in order to use these coefficients to infer out-of-sample estimates of costs. The majority of the Wald tests reported in Appendix 3 suggest that the relationship between ISS and LOS for our sample is not statistically different for the QTR populations of interest. Thus, we used the estimated parameters calculate an $e x$ post prediction of costs for 2004 .

To provide a contemporaneous estimate of cost the same methodology was applied to 2008 data. However, this was done only after first considering the potential impact that any technological change might have had on cost of treatment. The increased implementation of procedures such as non-operative management of solid organ injury, angiographic embolisation, and endovascular stenting, may potentially increase or decrease costs. ${ }^{\text {iv }}$ However, when LOS for inpatients treated for major trauma in Queensland in 2004 and 2008 (see Table 1) were compared, no statistical difference was found, which implies that in total the impact of technological change on LOS between 2004 and 2008 has probably not been great, at the level of the population of major trauma patients, in this jurisdiction.

Our economic approach, which used QTR data to mediate our sample cost estimates resulted to an adjustment from our sample mean of $\mathrm{A} \$ 102,851$ to A $\$ 68,419$ (Model 4) and A $\$ 74,664$ (Model 3) for the computation of our out-ofsample estimates. These results support the hypothesis that the cost of major trauma was significantly greater than has previously been documented. Doran et al. (2005) reported that the total pre-hospital and hospital costs of major trauma treated in Queensland from 1998 to 2001 were A\$19.5 million and A\$126.6 million, respectively. This represents an annualised cost of approximately A $\$ 36.55$ million or $A \$ 9,310$ per treated patient.(12) In the sample of inpatients treated at PAH in 2004, the direct cost of major trauma was $\mathrm{A} \$ 76,299$ per treated inpatient and the total clinical and non-clinical costs were estimated to be $\mathrm{A} \$ 102,851$ per treated inpatient. According to our (conservative) estimates, the true aggregate cost

\footnotetext{
${ }^{\text {iv }}$ We wish to thank an anonymous reviewer for making this point.
} 
of trauma was between $\mathrm{A} \$ 86.1$ million and $\mathrm{A} \$ 106.4$ million for the State of Queensland, in 2004.

Three factors explain our higher cost estimates. First, our bottom-up costing methodology captures clinical costs in the sub-sample of inpatients treated for major trauma, which cannot be fully captured using a mean DRG cost weight. Secondly, the inclusion of readmissions was found to increase the costs of inpatient care by a further $38.1 \%$. This finding has important implication for future economic evaluations that are designed to estimate the costs of trauma. Finally, the inclusion of $\mathrm{A} \$ 24,273$ in indirect costs incurred during the first year following discharge increased the final cost estimate by a further $30.9 \%$. Interestingly, including the costs of ambulatory care and pharmaceutical consumption did not have a particularly large effect on our cost estimates.

The 2008 cost estimates suggest that the nominal (real) costs of major trauma in Queensland have increased by $56.5 \%$ (38.5\%) since 2004. This cost increase, which appears in large part to be due to the increased numbers of inpatients being treated for major trauma in Queensland; (2004 = 1300 and $2008=$ $1832)^{\mathrm{v}}$, obviously will be of concern to policy-makers and practitioners. The accurate quantification of costs is an important initial step towards ensuring that people treated for major trauma receive an appropriate allocation of scarce medical resources. Our partial economic analysis makes a significant contribution by quantifying the costs incurred by this vulnerable sub-population of hospital inpatients. This paper provides two further contributions that are important in this regard. First, it demonstrates that a 'bottom-up' approach provides a more complete, and we would argue accurate, estimate of direct costs. The import of this is that such costing exercises - which themselves are more expensive to conduct should nevertheless be considered when evaluating sub-populations of inpatients that are believed not to be well-represented by the DRG cost-weights or other casebased financing formulae. Secondly, we demonstrate empirically that re-

\footnotetext{
${ }^{\mathrm{v}}$ Note however, that one additional hospital was added to the QTR in 2008.
} 
admissions for major trauma patients can be important drivers of hospital costs, even though they are not commonly measured. This finding not only has implications for the future cost evaluation of major trauma, but also for other inpatient cohorts that may require ongoing hospital treatment.

\section{ACKNOWLEDGEMENTS}

The Human Research and Ethics Committee (HREC) at PAH granted this study ethical approval on the 7th of April 2006, with the condition that the sample be cross-matched with the Death Registry in Queensland to avoid contact with the families of deceased patients. The University of Queensland granted an expedited ethical approval based upon the approval of the PAH HREC. MBS and PBS data from January 2004 until December 2005 were extracted with individual patient consent, using a Medicare Consent Form.

Funding was provided by the Royal Australasian College of Surgeons for the Trauma Fellowship 2006 and is gratefully acknowledged. Funding provided by the Motor Accident Insurance Commission, via the Centre for Economic Research on Health is also gratefully acknowledged. The authors would also thank the staff of the Princess Alexandra Hospital and Queensland Ambulance Service for providing data and assistance to the research team. 


\section{BIBLIOGRAPHY}

1. Mathers C, Penn R. Health system costs of injury, poisoning and musculoskeletal disorders in Australia 1993-94. AIHW cat. no. HWE 12. Canberra: Australian Institute of Health and Welfare (Health and Welfare Expenditure Series no. 6), 1999.

2. Walsh J. Costs of Spinal Cord Injury in Australia. Paraplegia. 1988;26:3808.

3. Rowell D, Connelly LB. Personal assistance, income and employment: the spinal injuries survey instrument (SISI) and its application in a sample of people with quadriplegia. Spinal Cord. 2008;46(6):417-24.

4. Randell A, Sambrook PN, Nguyen TV, Lapsley H, Jones G, Kelly PJ, et al. Direct clinical and welfare costs of osteoporotic fractures in elderly men and women. Osteoporosis International. 1995;5(6):427-32.

5. Sanders KM, Nicholson GC, Ugoni AM, Pasco JA, Seeman E, Kotowicz MA. Health burden of hip and other fractures in Australia beyond 2000 Projections based on the Geelong Osteoporosis Study MJA 1999;170:467-70.

6. Connelly LB, Supangan R. The economic costs of road traffic crashes: Australia, states and territories. Accident Analysis and Prevention. 2006;38(6):1087-93.

7. Small TJ, Sheedy JM, Grabs AJ. Cost, demographics and injury profile of adult pedestrian trauma in inner Sydney ANZ Journal of Surgery. 2006;76(1/2):437.

8. Moorin RE, Hendrie D. The epidemiology and cost of falls requiring hospitalisation in children in Western Australia: A study using linked administrative data. Accident Analysis \& Prevention. 2008;40(1):216-22.

9. Hall SE, Hendrie D. A prospective study of the costs of falls in older adults living in the community. Australian and New Zealand Journal of Public Health. 2003;27(3):343-51.

10. Bradely C, Harrison JE. Hospitalisations due to fall in older people, Australia, 2003/04. Adelaide: AIHW, 2007.

11. Hendrie D. The cost of injury in Western Australia during 2003: An overview of total health system costs. Perth, Western Australia Western Australian Government 2005.

12. Doran C, Gasgoine M, Petrie D. The cost of major trauma: Queensland trauma plan project (sub-study 5). In: Tippett V, FitzGerald G, Pollard C, Clark M, Geraghty T, Aitken L, et al., editors. Queensland Trauma Plan Project 2001-2005. Brisbane: Australian Centre for Prehospital Research; 2006.

13. Watson WL, Ozanne-Smith J. The Cost of Injury to Victoria. Melbourne Monash University Accident Research Centre, 1997124. 
14. Moller J. Estimated cost of injury (\$millions) by all causes Australia 199596. Research Centre for Injury Studies 1998.

15. Hendrie D, Legge M. Interim report on the costs of Injury database for Western Australia. Perth Injury Research Centre, 2003 July 2003. Report No.

16. Dallow N, Mason D, Ward j, Kefu L, Lang J, Bellamy N. Queensland Trauma Registry: A summary of injuries treated at Princess Alexandra Hospital 2005. Brisbane Centre of National Research on Disability and Rehabilitation Medicine, 2007 2007. Report No.

17. Osler T, Baker SP, Long W. A modification of the injury severity score that both improves accuracy and simplifies scoring. Journal of Trauma-Injury Infection \& Critical Care. 1997;43(6):922.

18. Australia. Dept. of Health and Ageing. Australian refined diagnosis related groups : version 5.1 : Definitions manual. Canberra: Commonwealth Dept. of Health and Ageing; 2004.

19. Webber J, Connelly L. Cost of Trauma Instrument (COTI) Brisbane 2005 [06/2009]; Available from: http://www.uq.edu.au/conrod/.

20. Drummond MF, O'Brien BJ, Stoddart GL, Torrence GW. Methods for the Economic Evaluation of Health Care Programs. 2nd ed. New York: Oxford University Press; 1998.

21. Andrews M. (Eclipsys Corporation), email. In: Rowell D, editor. 2009.

22. Azoulay A, Doris N, Filion K, Caron J, Pilote L, Eisenberg M. The use of the transition cost accounting system in health services research. Cost Effectiveness and Resource Allocation. 2007;5(1):11.

23. Queensland Health. Quarterly public hospitals performance report December quarter 2009. Brisbane Queensland Department of Health 2009.

24. Stevenson M, Segui-Gomez M, Lescohier I, Di Scala C, McDonald-Smith G. An overview of the injury severity score and the new injury severity score. British Medical Journal. 2001;7(1):10-3.

25. Commonwealth Department of Health and Ageing in conjunction with the States and Territories. National Hospital Cost Data Collection V5.0 Public Sector Cost Weights for AR-DRG Version 5.0, Round 9 (2004-05) Queensland. Canberra DoHA, 2006.

26. Chapko MK, Liu CF, Perkins M, Li YF, Fortney JC, Maciejewski ML. Equivalence of two healthcare costing methods: bottom-up and top-down. Health Economics. 2008. 


\section{APPENDICIES}

Appendix 1: Admission for Major trauma by AR-DRG V5.0

\begin{tabular}{|c|c|c|c|}
\hline Admissions for Major Trauma by AR-RDG V5.0 & $\begin{array}{c}\text { No. } \\
\text { Admits }\end{array}$ & $\begin{array}{l}\text { No. Re- } \\
\text { admits }\end{array}$ & $\begin{array}{c}\text { Cost } \\
\text { Weights } \\
(25) \\
\end{array}$ \\
\hline W01Z - Ventilation or Craniotomy Procedures for Multiple Significant Trauma & 12 & 8 & 22.92 \\
\hline W02Z - Hip, Femur and Limb Procedures for Multiple Significant Trauma, incl Implantation & 3 & 2 & 8.48 \\
\hline W03Z - Abdominal Procedures for Multiple Significant Trauma & 2 & 1 & 6.27 \\
\hline W04Z - Other O.R. Procedures for Multiple Significant Trauma & 3 & 3 & 8.37 \\
\hline W61Z - Multiple Trauma Without Significant Procedures & 10 & 2 & 3 \\
\hline Totals & 30 & 16 & \\
\hline
\end{tabular}

Appendix 2: Readmissions for Ongoing Rx by AR-RDG V5.0

Readmissions for Ongoing Rx by AR-RDG V5.0

No. Re-admits by Dx.

901Z - Extensive O.R. Procedure Unrelated to Principal Diagnosis

D12Z - Other Ear, Nose, Mouth \& Throat Procedures

G08Z - Abdominal, Umbilical and Other Hernia Procedures Age $>0$

I08B - Other Hip and Femur Procedures W/O Catastrophic or Severe CC

I12C - Infect/Inflam Bone \& Joint W Misc Musc Sys \& Conn Tiss Proc W/O Cat or Sev CC

I23Z - Local Excision \& Removal of Internal Fixation Device Excl Hip and Femur

I27B - Soft Tissue Procedures W/O Catastrophic or Severe CC

I64B - Osteomyelitis W/O CC

I73C - Aftercare of Connective Tissue Disorders Age $<60$ W/O Catastrophic or Severe CC

X06B - Other Procedures for Other Injuries W/O Catastrophic or Severe CC

Z01B - O.R. Procedures W Diagnoses Other Contacts W Health Services W/O Cat/Sev CC

Z60A - Rehabilitation W Catastrophic or Severe CC

Z60B - Rehabilitation W/O Catastrophic or Severe CC

Z62Z - Follow Up After Completed Treatment W/O Endoscopy

Totals

1
1
1
1
1
3
3
1
2
1
1
8
2
5
31

Source: Health Information Management Services PAH 
Appendix 3 Wald Tests for Equality of Sample and Population ISS Coefficients; using OLS and Poisson regression

\begin{tabular}{|c|c|c|c|c|c|c|c|c|}
\hline \multirow[t]{2}{*}{ Models } & \multicolumn{4}{|c|}{ OLS Regression } & \multicolumn{4}{|c|}{ Poisson Regression } \\
\hline & $\begin{array}{l}\text { Constant } \\
\text { (S.E) }\end{array}$ & $\begin{array}{l}\text { ISS } \\
\text { (S.E) }\end{array}$ & $\mathrm{R}^{2}$ & $\begin{array}{c}\text { Wald tests for equality } \\
\text { of } \\
\text { ISS coefficients }\end{array}$ & $\begin{array}{l}\text { Constant } \\
\text { (S.E) }\end{array}$ & $\begin{array}{l}\text { ISS } \\
\text { (S.E) }\end{array}$ & $\mathrm{R}^{2}$ & $\begin{array}{c}\text { Wald tests for equality } \\
\text { of } \\
\text { ISS coefficients }\end{array}$ \\
\hline $\begin{array}{l}\text { Model } 1 \\
\text { PAH } 2004(n=30)\end{array}$ & $\begin{array}{l}-33.96 \\
(23.17) \\
\end{array}$ & $\begin{array}{c}* * 2.36 \\
(0.78)\end{array}$ & 0.247 & n.a. & $\begin{array}{c}* * * 1.81 \\
(0.09) \\
\end{array}$ & $\begin{array}{l}* * * 0.05 \\
(0.002) \\
\end{array}$ & 0.269 & n.a. \\
\hline $\begin{array}{l}\text { Model } 2 \\
\text { PAH } 2004(\mathrm{~N}=326)\end{array}$ & $\begin{array}{c}6.39 \\
(4.29)\end{array}$ & $\begin{array}{c}* * * 0.66 \\
(0.16)\end{array}$ & 0.052 & $\begin{array}{l}\mathrm{H}_{0}: \mathrm{ISS}_{\text {Model1 }}-\mathrm{ISS}_{\text {Model } 2} \\
=0 \\
\text { Chi2 }(1)=2.21 \\
\text { Prob. }>\text { chi2 }=0.1371 \\
\text { Do not reject the null } \\
(90 \% \mathrm{Cl})\end{array}$ & $\begin{array}{c}* * * 2.53 \\
(0.03)\end{array}$ & $\begin{array}{l}* * * 0.02 \\
(0.001)\end{array}$ & 0.057 & $\begin{array}{l}\mathrm{H}_{0}: I S S_{\text {Model } 1}-\text { ISS }_{\text {Model }} \\
2=0 \\
\text { Chi2 }(1)=2.91 \\
\text { Prob. > chi2 }=0.0882 \\
\text { Reject the null } \\
(90 \% \mathrm{Cl})\end{array}$ \\
\hline $\begin{array}{l}\text { Model } 3 \\
\text { QLD } 2004(N=1300)\end{array}$ & $\begin{array}{c}* * * 5.64 \\
(1.56)\end{array}$ & $\begin{array}{c}* * * 0.48 \\
(0.06)\end{array}$ & 0.049 & $\begin{array}{l}\mathrm{H}_{0}: \mathrm{ISS}_{\text {Model1 }}-\mathrm{ISS}_{\text {Model } 3} \\
=0 \\
\mathrm{Chi} 2(1)=2.79 \\
\text { Prob. }>\text { chi2 }=0.0948 \\
\text { Reject the null }(90 \% \mathrm{Cl})\end{array}$ & $\begin{array}{c}* * * 2.31 \\
(0.02)\end{array}$ & $\begin{array}{l}* * * 0.02 \\
(0.001)\end{array}$ & 0.049 & $\begin{array}{l}\mathrm{H}_{0}: \mathrm{ISS}_{\text {Model1 }}-\mathrm{ISS}_{\text {Model }} \\
3=0 \\
\mathrm{Chi} 2(1)=3.56 \\
\text { Prob. }>\text { chi2 }=0.0594 \\
\text { Reject the null } \\
(90 \% \mathrm{Cl})\end{array}$ \\
\hline $\begin{array}{l}\text { Model } 4 \\
\text { PAH } 2008(\mathrm{~N}=440)\end{array}$ & $\begin{array}{l}*-6.58 \\
(3.49)\end{array}$ & $\begin{array}{l}* * * 1.07 \\
(0.13)\end{array}$ & 0.129 & $\begin{array}{l}\mathrm{H}_{0}: I S S_{\text {Model1 }}-I_{\text {ISS }} \text { Model } 4 \\
=0 \\
\text { Chi2(1) }=1.26 \\
\text { Prob. }>\text { chi2 }=0.2608 \\
\text { Do not reject the null } \\
(90 \% \mathrm{Cl})\end{array}$ & $\begin{array}{l}* * * 1.98 \\
(0.03)\end{array}$ & $\begin{array}{l}{ }^{* * *} 0.04 \\
(0.001)\end{array}$ & 0.137 & $\begin{array}{l}\mathrm{H}_{0}: \mathrm{ISS}_{\text {Model } 1}-\mathrm{ISS}_{\text {Model }} \\
4=0 \\
\mathrm{Chi} 2(1)=0.79 \\
\text { Prob. > chi2 }=0.3755 \\
\text { Do not reject the null } \\
(90 \% \mathrm{Cl})\end{array}$ \\
\hline $\begin{array}{l}\text { Model } 5 \\
\text { QLD } 2008(\mathrm{~N}=1832)\end{array}$ & $\begin{array}{l}{ }^{*} 2.80 \\
(1.45)\end{array}$ & $\begin{array}{c}* * * 0.56 \\
(0.06)\end{array}$ & 0.052 & $\begin{array}{l}\mathrm{H}_{0}: \mathrm{ISS}_{\text {Model1 }}-\mathrm{ISS}_{\text {Model } 5} \\
=0 \\
\text { Chi2(1) }=2.57 \\
\text { Prob. }>\text { chi2 }=0.1086 \\
\text { Do not reject the null } \\
(90 \% \mathrm{Cl})\end{array}$ & $\begin{array}{c}* * * 2.12 \\
(0.01)\end{array}$ & $\begin{array}{l}{ }^{* * *} 0.02 \\
(0.001)\end{array}$ & 0.056 & $\begin{array}{l}\mathrm{H}_{0}: I S S_{\text {Model } 1}-\text { ISS }_{\text {Model }} \\
5=0 \\
\text { Chi2 }(1)=2.51 \\
\text { Prob. > chi2 }=0.1131 \\
\text { Do not reject the null } \\
(90 \% \mathrm{Cl})\end{array}$ \\
\hline
\end{tabular}

Source: (i) Health Information Management Services PAH, (ii) Medicare Statistics Australia and (iii) the COTI (iv) QTR 